\title{
MANIFESTATION OF A COEXISTENCE OF THE LONG-PERIODIC PHASE WITH THE INCOMMENSURATE PHASE
}

\author{
S. Sveleba, I. Poloviniko, V. Zhmurko \\ Physical Department, State University, Dragomanova st. 19, Lviv, 290005, Ukraine \\ AND Y. PanKIVSKYi
}

Department of Ecology, Ukrainian State University of Forestry and Wood Technology O. Kobylyanskoyi st. 1, Lviv, 290005, Ukraine

(Received July 27, 1998; revised version January 6, 1999; in final form May 5, 1999)

Electric and optical properties of the commensurate ferroelectric long-periodic phase in $\left[\mathrm{N}\left(\mathrm{CH}_{3}\right)_{4}\right]_{2} \mathrm{ZnCl}_{4}$ and $\left[\mathrm{N}\left(\mathrm{CH}_{3}\right)_{4}\right]_{2} \mathrm{CoCl}_{4}$ crystals were investigated to explain mechanism of the double hysteresis loops appearance.

PACS numbers: $64.70 .-p$

\section{Introduction}

It has been found that the presence of the double dielectric hysteresis loops in the commensurate regions manifests a coexistence of the long-periodic phases within-the inconmensurate phase. Such phenomenon was already investigated in a few papers $[1,2]$. Double hysteresis loops have been observed just above $T_{c}$ within the incommensurate phase on $\varepsilon=f(E)$ dependences in $\left[\mathrm{NH}_{4}\right] \mathrm{BeF}_{4}$ and $\mathrm{Rb}_{2} \mathrm{ZnCl}_{4}$ crystals. In accordance with the data of [3] the appearance of double hysteresis loops in the crystal has been explained on the basis of the defect density wave (DDW) model. It was shown that antipolarised regions disappear under influence of electric field and left the vestige, which serves as a germ at field removal. This vestige may be considered as residual defects concentration around soliton [4], that actually causes DDW existence in the crystal. DDW period coincides with the incommensurate structure period at the field absence.

The phase induced by the electric field in the incommensurate phase consists of regular clomain-like regions $D_{1}^{+}\left|D_{2}^{-}\right| D_{3}^{+}\left|D_{4}^{-}\right| D_{5}^{+}\left|D_{6}^{-}\right| D_{1}^{+} \mid \ldots$, where neighbouring domains $D_{i}, D_{i+1}$ with opposite direction of spontaneous polarization $P_{\mathrm{s}}$ are divided by domain walls $W_{i, i+1}$. Under the electric field influence the width of 
positively oriented domains increases and for electric field strong enough the negatively oriented domains may disappear. But to retain its own phase change the crystalline structure does not become totally uniform. It consists rather of regular parts $D_{1}^{+}, D_{3}^{+}, D_{5}^{+}, D_{1}^{+}$, divided by boundaries $W_{13}, W_{35}, W_{51}$ with opposite phases. When the electric field is switched off, the walls are widened to the size of the normal domain walls, and the original non-polarized state reappears. It should also be noted that analogous dielectric hysteresis loops arise in the commensurate regions of the incommensurate phase lying far from the phase transitions. For example, similar hysteresis loops were observed in such regions of betaine calcium chloride dihydrate $\left(\mathrm{CH}_{3}\right)_{3} \mathrm{NCH}_{2} \mathrm{COO} \cdot \mathrm{CaCl}_{2} \cdot 2 \mathrm{H}_{2} \mathrm{O}$ (BCCD) crystal [5].

In principle, two explanations of this phenomenon should be considered. The first one is comprised with transformation into single domain state of the soliton structure under field influence in the commensurate regions (where the soliton structure possesses a domain-like character of the commensurate region). The second explanation is related to the coexistence of the commensurate long-periodic phase with the incommensurate phase and disappearance of one of them under the influence of electric field. Experimental investigations of electrooptic properties in the commensurate ferroelectric long-periodic phases of $\left[\mathrm{N}_{(}\left(\mathrm{CH}_{3}\right)_{4}\right]_{2} \mathrm{ZnCl}_{4}$ and $\left[\mathrm{N}\left(\mathrm{CH}_{3}\right)_{4}\right]_{2} \mathrm{CoCl}_{4}$ crystals were performed for explanation of the mechanism of double hysteresis loops appearance.

The crystals were grown by the slow evaporation of an aqueous solution of the $\left[\mathrm{N}\left(\mathrm{CH}_{3}\right)_{4}\right]_{2} \mathrm{Cl}$ and $\mathrm{ZnCl}_{2}$ salts, taken in the proper stoichiometric ratios. Changes of the birefringence $\delta\left(\Delta n_{i}\right)$ under the influence of electric field of strength $E$ were measured by Senarmonth's method [6]. The temperature was stabilised with an accuracy of $\pm 0.005 \mathrm{~K}$.

\section{Experimental results and discussion}

The experimental field dependences of $\delta\left(\Delta n_{i}\right)$ in the commensurate ferroelectric long-periodic phase of $\left[\mathrm{N}\left(\mathrm{CH}_{3}\right)_{4}\right]_{2} \mathrm{ZnCl}_{4}$ crystals are presented in Fig. 1 . One can see that optical birefringence $\delta(\Delta n)$ shows anomalous behaviour under the influence of electric field. The obtained dependences correlate with stress and temperature dependences of $\delta\left(\Delta n_{i}\right)$ (obtained in conditions of the "viscous" interaction). The latter according to [7] was explained in terms of a change of soliton density under the action of external forces. At small field strength $\left(0 \leq E \leq 6 \times 10^{3} \mathrm{~V} / \mathrm{m}\right)$ double electrooptic hysteresis loops with coercive field strength $E_{\mathrm{c}} \approx 2 \times 10^{3} \mathrm{~V} / \mathrm{m}$ are observed (Fig. 2). They possess an analogous character as the loops observed in $\left[\mathrm{N}\left(\mathrm{CH}_{3}\right)_{4}\right]_{2} \mathrm{CoCl}_{4}[8], \mathrm{BCCD}[5],\left[\mathrm{NH}_{4}\right]_{2} \mathrm{BeF}_{4}$ [1] crystals.

First of all, let us consider a phenomenological description of the obtained results. The first motion integral in the case of the electric field of strength $E_{1}$ applied to the sample may be written according to [9]:

$$
\frac{1}{2}\left(\frac{\mathrm{d} \varphi}{\mathrm{d} z}\right)^{2}-\frac{\alpha_{l}^{\prime} \rho^{2 l-2}}{2 \gamma} \cos 2 l \varphi+\frac{a_{1}}{\gamma} E_{1} \rho^{l} \cos l \varphi=\Xi,
$$

where $\rho$ and $\varphi$ denote the amplitude and phase of the two-component order parameter $\eta_{1}=\rho \cos \varphi$ and $\eta_{2}=\rho \sin \varphi$, respectively; $\alpha_{l}^{\prime}, a_{1}, \gamma$ are expansion coefficients; 


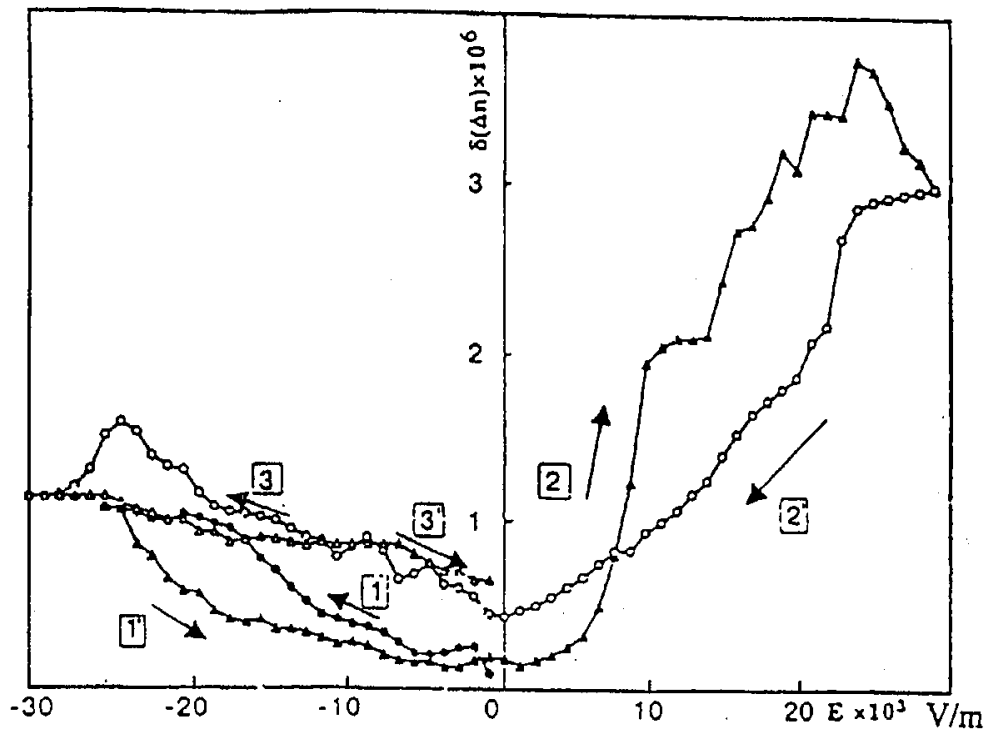

Fig. 1. Dependence of $\delta(\Delta n)$ on applied electric field $E$ for $\left[\mathrm{N}\left(\mathrm{CH}_{3}\right)_{4}\right] \mathrm{ZnCl} \mathrm{nl}_{4}$ crystal at $T=282 \mathrm{~K}: 1-1^{\prime}, 2-2^{\prime}, 3-3^{\prime}-$ field cycles, the arrows show direction of the changes.

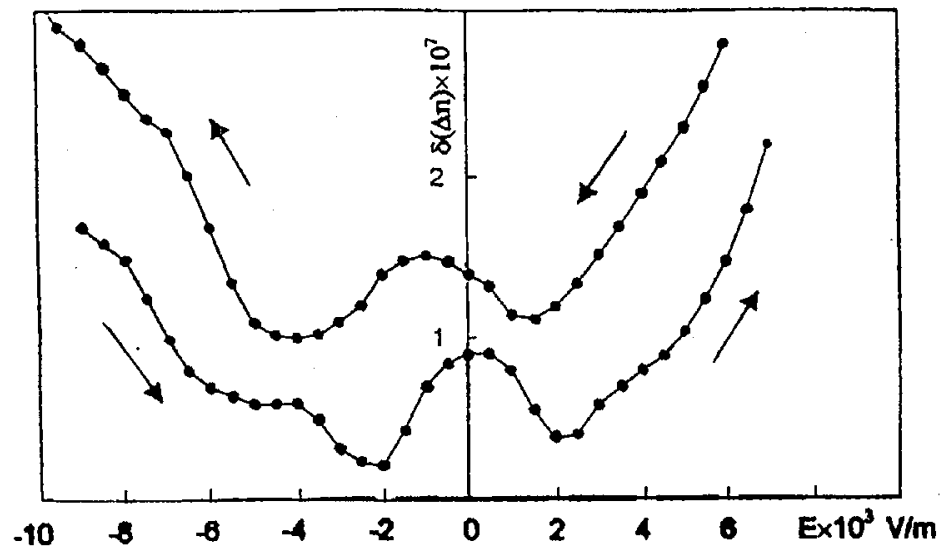

Fig. 2. Electrooptic loop in the $\delta(\Delta n)=f(E)$ co-ordinates measured in the region of

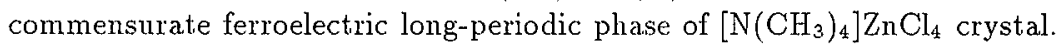

$\rho^{l}$ comes from Fourier's transformation of the thermodynamical potential; $\Xi$ is the integrating constant. Let us rewrite Eq. (1) in the form

$$
\frac{\mathrm{d} \varphi}{\mathrm{d} z}=\left(\Xi+\frac{\alpha_{l}^{\prime} \rho^{2 l-2}}{\gamma} \cos 2 l \varphi-\frac{2 a_{1} E_{1} \rho^{l}}{\gamma} \cos l \varphi\right)^{1 / 2} .
$$

Consequently for the $\delta\left(\Delta n_{i}\right)$ we obtain the following expression:

$$
\delta\left(\Delta n_{i}\right) \approx \nu_{i j} \frac{1}{d} \rho^{2} \int_{0}^{d}\left(\Xi+\frac{\alpha_{l}^{\prime} \rho^{2 l-2}}{\gamma} \cos 2 l \varphi-\frac{2 a_{1} E_{1} \rho^{l}}{\gamma} \cos l \varphi\right)^{1 / 2} \mathrm{~d} z,
$$

where $\nu_{i j}$ is the expansion coefficient; $d$ denotes the sample thickness. 
In accordance with the data of Ref. [10] the change of the phase of order parameter could be presented as

$$
\varphi=\varphi_{0}+\varphi_{1}, \quad \text { where } \quad \varphi_{1}=\frac{\mathrm{d} \varphi_{0}}{\mathrm{~d} z} \int_{0}^{z} \frac{a E_{1} \rho^{l} \sin l \varphi_{0}+C}{2 \gamma\left(\mathrm{d} \varphi_{0} / \mathrm{d} z\right)^{2}} \mathrm{~d} z
$$

$C$ denotes the integrating constant; $\varphi_{0}$ does not depend on $E_{1}$.

At first approximation it is possible to assume that $\varphi_{1} \approx b_{1} E_{1}$, where $b_{1}$ is the expansion coefficient [10]. Hence, the $\delta\left(\Delta n_{i}\right)$ may be expressed as

$$
\begin{aligned}
& \delta\left(\Delta n_{i}\right) \approx \nu_{i j} \frac{1}{d} \rho^{2} \\
& \times \int_{0}^{d}\left[\Xi+\frac{\alpha_{l}^{\prime} \rho^{2 l-2}}{\gamma} \cos 2 l\left(\varphi_{0}+b_{1} E_{1}\right)-\frac{2 a_{1} E_{1} \rho^{l}}{\gamma} \cos l\left(\varphi_{0}+b_{1} E_{1}\right)\right]^{1 / 2} \mathrm{~d} z
\end{aligned}
$$

Taking into account that spontaneous polarisation in these commensurate regions appears perpendicularly to the direction of incommensurate modulation and $E_{1}=E_{x}$, Eq. (5) could be rewritten as

$$
\begin{aligned}
& \delta\left(\Delta n_{i}\right) \approx \nu_{i j} \frac{1}{d} \rho^{2} \\
& \times \int_{0}^{d}\left[\Xi+\frac{\alpha_{l}^{\prime} \rho^{2 l-2}}{\gamma} \cos 2 l\left(\varphi_{0}+b_{1} E_{x}\right)-\frac{2 a_{1} E_{1} \rho^{l}}{\gamma} \cos l\left(\varphi_{0}+b_{1} E_{x}\right)\right]^{1 / 2} \mathrm{~d} x \\
& =\nu_{i j} \rho^{2}\left[\Xi+\frac{\alpha_{l}^{\prime} \rho^{2 l-2}}{\gamma} \cos 2 l\left(\varphi_{0}+b_{1} E_{x}\right)-\frac{2 a_{1} E_{x} \rho^{l}}{\gamma} \cos l\left(\varphi_{0}+b_{1} E_{x}\right)\right]^{1 / 2},
\end{aligned}
$$

since the expression under the integral depends on the change of phase along the modulation axis. Field dependence of $\delta\left(\Delta n_{i}\right)$ obtained from (6) with the parameters corresponding to average values of the coefficients of the termodynamical potential [11] at $\rho=$ const is plotted in Fig. 3. It correlates with conformable experimental dependence. One can conclude from expression (6) that in the condition when energy of the electric field applied to the crystal will be equal to the energy of soliton lattice,

$$
\begin{aligned}
& \Xi-\frac{\alpha_{1}^{\prime} \rho^{2 l-2}}{\gamma}+\frac{2 a_{1} E_{1} \rho^{l}}{\gamma}=0, \quad \text { where } \quad \cos 2 l\left(\varphi_{0}+b_{1} E_{1}\right)=-1 ; \\
& \cos l\left(\varphi_{0}+b_{1} E_{1}\right)=a_{1} E_{1} /\left|a_{1} E_{1}\right|=1 ; \quad E_{\mathrm{c}}=E_{1},
\end{aligned}
$$

the domains of the commensurate ferroelectric long-periodic phase will be repolarised. Hence,

$$
E_{1}=E_{\mathrm{c}}=\frac{\alpha_{l}^{\prime} \rho^{l-2}}{a_{l}} .
$$

To clarify the mechanism of the double loops appearance let us consider the energy of soliton lattice as [9]

$$
\frac{\Phi}{2 \gamma \rho^{2} V}=\left(\frac{4 \sqrt{v}}{\pi}-\left|k_{0}\right|\right) \bar{k}+U(k)
$$




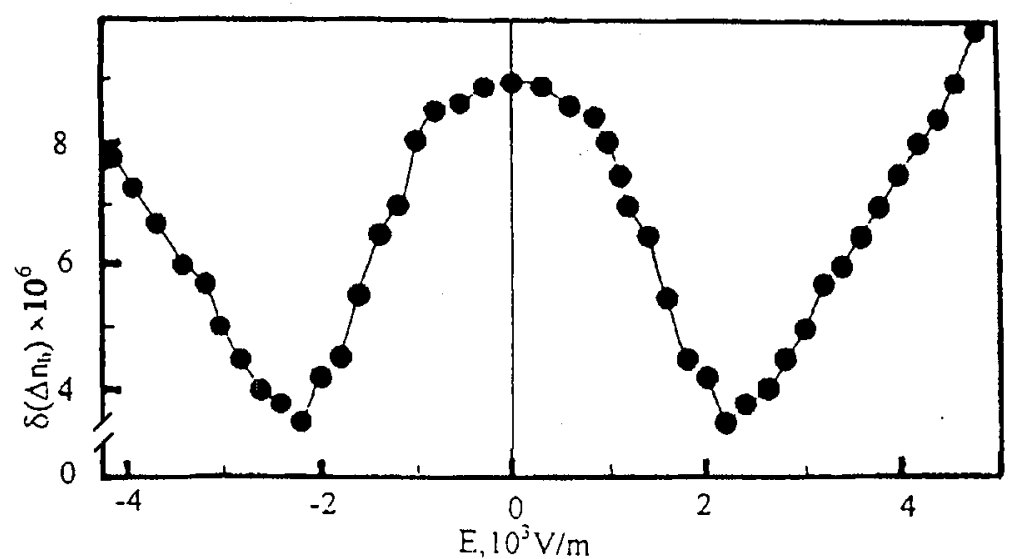

Fig. 3. Electric field dependence $\delta(\Delta n)$ calculated on the basis of Eq. (6) for $\left[\mathrm{N}\left(\mathrm{CH}_{3}\right)_{4}\right] \mathrm{ZnCl}_{4}$ crystal.

where $v=\alpha_{l}^{\prime} \rho^{2 l-2} / \gamma ; V$ denotes the volume of the sample; $k_{0}$ is the wave vector of the incommensurately modulated structure; $\bar{k}$ is the average wave vector of the soliton structure; $U(k)$ denotes the soliton repulsion energy. In accordance with expression (8) at $\left|k_{0}\right|=k_{0}^{*}$, where

$$
k_{0}^{*}=\frac{4 \rho^{l-1}}{\pi} \sqrt{\frac{\alpha_{i}^{\prime}}{\gamma}},
$$

there will occur the transition from the heterogeneous to homogeneous state [9]. In this case, the expression (9) according to (7) may be presented in the form

$$
k_{0}^{*}=\frac{4 E_{k} \rho a_{1}}{\pi \sqrt{\alpha_{l}^{\prime} \gamma}} .
$$

At low electric field one has $k_{0}^{*}-\left|k_{0}\right|<0$ and the availability of the solitons is preferable for the system. When this expression gets a positive value (at $E_{1}=E_{c}$ ) the solitons will disappear. Hence, at $E_{1}=E_{\mathrm{c}}$ the solitons in a sample disappear and the transition from the heterogeneous state to the homogeneous one occurs.

To confirm the transition, we studied the electric field influence on temperature behaviour of the optical indicatrix rotation angle in the commensurate long-periodic phase. The obtained experimental data for $\left[\mathrm{N}\left(\mathrm{CH}_{3}\right)_{4}\right]_{2} \mathrm{ZnCl} \mathrm{Cl}_{4}$ crystal are presented in Fig. 4. As one can see, at the absence of the electric field the $\varphi_{\alpha}$ value smoothly varies with temperature and shows inessential anomalous changes. Electric field of strength $E=10^{4} \mathrm{~V} / \mathrm{m}$ applied to the sample results in an anomalous temperature behaviour of $\varphi_{a}$. The optical indicatrix rotation (Fig. 4) is observed only in the commensurate homogeneous long-periodic phase (so far as $E=10^{4}>E_{\mathrm{c}}$ ). Let us note that in this case according to the data of [12] the rotation of the optical indicatrix arises at the electric field $E$ applied along $b$-axis. Thus, in the commensurate long-periodic phases the transition from the heterogeneous to the homogeneous state with disappearance of the soliton structure occurs a.t the field $E=E_{c}$. 


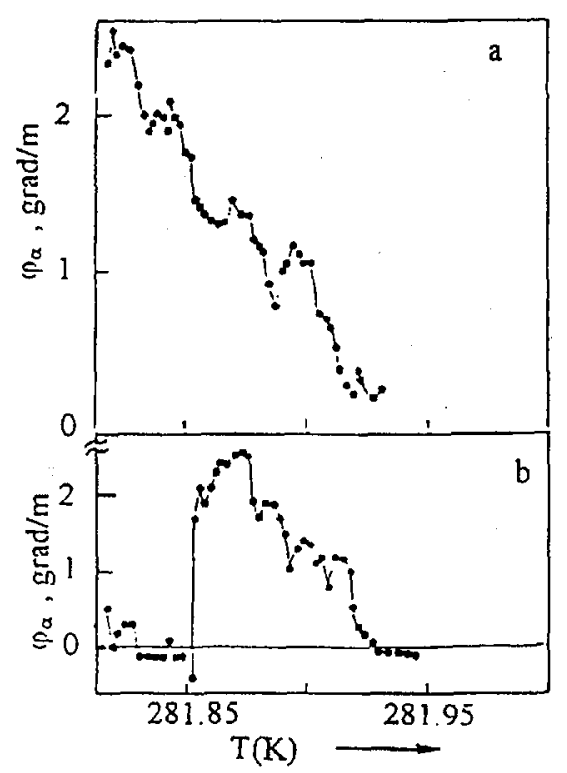

Fig. 4. Temperature dependence of the optical indicatrix rotation angle $\varphi_{\alpha}$ for $\left[\mathrm{N}\left(\mathrm{CH}_{3}\right)_{4}\right] \mathrm{ZnCl}_{4}$ crystal in the incommensurate phase at $E=0(\mathrm{a})$ and $E=10^{4} \mathrm{~V} / \mathrm{m}(\mathrm{b})$.

Therefore, the incommensurate phase and the commensurate ferroelectric one coexist in the commensurate ferroelectric long-periodic phase. Let us consider this coexistence in detail. It has been found that in the commensurate long-periodic phase the incommensurability wave vector takes a commensurate value $m / n$. Thus, $m$ unit cells are packed into $n$ periods of the modulation wave. Hence, $n$ periods of the incommensurability waves (where $n$ corresponds to the definite $m$ value) will be contained in one commensurate domain (for example, ferroelectric domain). The neighbouring domain will contain the same number of solitons, but spontaneous polarisation or deformation will get an opposite sign. A schematic picture of this coexistence is shown in Fig. 5.

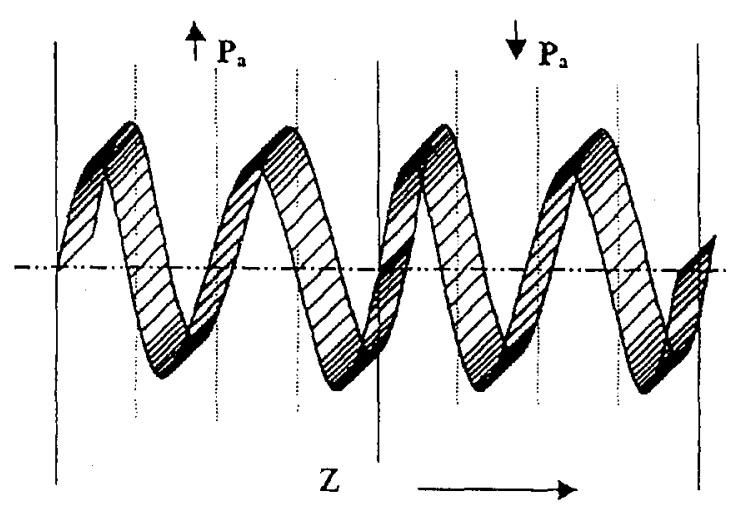

Fig. 5. The schema of the commensurate phase coexistence with the incommensurate structure. The solid lines correspond to domain walls, the dash lines - to solitons. 
Let us discuss the results of investigations of the dielectric properties of $\left[\mathrm{N}\left(\mathrm{CH}_{3}\right)_{4}\right]_{2} \mathrm{CoCl}_{4}$ crystals to corroborate the considered model of phases coexistence [8]. Double hysteresis loops were observed clearly at $E=E_{\mathrm{c}}=5 \times 10^{3} \mathrm{~V} / \mathrm{m}$ inside the commensurate long-periodic phase at $T=281 \mathrm{~K}$ on the field dependences of dielectric permittivity $\varepsilon$. When approaching the phase transition temperatures $T_{2}=280.4 \mathrm{~K}$ and $T_{3}=281.1 \mathrm{~K}$ the $E_{\mathrm{c}}$ value decreases and the hysteresis loop varies from the double loop to the constricted loop. To explain the obtained results let us analyse expression (7). As one can see, the coercive field will increase when the value of the order parameter (namely, of spontaneous polarisation $P_{\mathrm{s}}$ ) decreases. Therefore, when approaching the phase transition temperatures $T_{2}$ and $T_{3}$, the $P_{\mathrm{s}}$ value decreases, consequently the coercive field value will be also diminished that was observed in experiment. When the value of energy of soliton appearance is equal to the energy of soliton pinning on defects, the hysteresis double loop will transform into a constricted loop. It is necessary to note that at $E=E_{\mathrm{c}}$ the energy $W$ of the capacitor (investigated sample with electrodes) will be equal to the energy of soliton lattice at $E=0: W_{\text {sol }}=0.797 \times 10^{-3} \mathrm{~J}$ (at $T=280.4 \mathrm{~K}$ ) and $W_{\text {sol }}=1.022 \times 10^{-3} \mathrm{~J}$ (at $T=281 \mathrm{~K}$ ). The difference of the $W_{\text {sol }}$ values at various temperatures in the commensurate ferroelectric long-periodic phase testifies to the occurrence of the interaction between the commensurate and the incommensurate phases.

If we suppose that the incommensurate phase is a set of the successive long-periodic commensurate phases [13], one should consider the interaction of such two phases as a particular case of the interaction between the commensurate and the incommensurate phases. Both experimental and calculated temper-

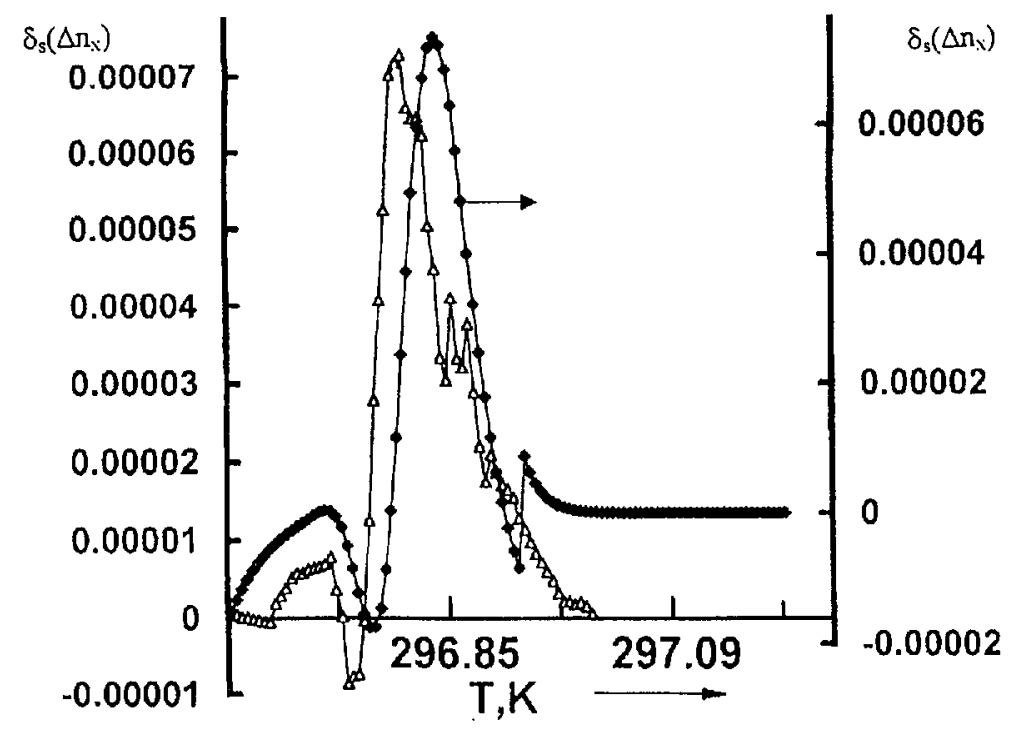

Fig. 6. Temperature dependence of optical birefringence along $a$-axis for $\left[\mathrm{N}\left(\mathrm{CH}_{3}\right)_{4}\right]_{2} \mathrm{CuCl}_{4}$ crystal at a rate of temperature change $\partial T / \partial t=60 \mathrm{mK} / \mathrm{h}$, $\Delta$ - experimental curve, - theoretical curve. 
ature dependences of $\delta(\Delta n)$ according to (3) (with the method described in [13]) for $\left[\mathrm{N}\left(\mathrm{CH}_{3}\right)_{4}\right]_{2} \mathrm{CuCl}_{4}$ crystals clearly reflect this interaction (Fig. 6). The latter manifests itself in anomalies of the birefringence.

\section{Conclusions}

The commensurate and the incommensurate phases coexist and interact in the commensurate long-periodic phase causing the double hysteresis loops appearance in co-ordinates $\varepsilon=f(E), \delta\left(\Delta n_{i}\right)=f(E)$. The question of existence of the boundaries with opposite phases and consequently the mechanism of DDW creation are still unclear. It is necessary to perform experimental investigation of the birefringence and dielectric properties at the transitional regions under electric field influence for detailed explanation of the considered interaction.

\section{References}

[1] B.A. Strukov, V.M. Arutyunova, I. Uesu, Fiz. Tverd. Tela 24, 3061 (1982).

[2] K. Deguchi, J. Crystallogr. Soc. Jpn. 30, 210 (1988).

[3] B.A. Strukov, P.S. Sminov, S.A. Taraskin, Y. Uesu, Ferroelectrics Lett. 11, 147 (1984).

[4] D.J. Srolovitz, R. Eykholt, D.M. Barnett, J.P. Hirth, Phys. Rev. B 35, 6107 (1987).

[5] S. Sveleba, V. Kapustianik, I. Polovinko, M. Bublyk, Z. Czapla, R. Strykowes, Phys. Status Solidi A 147, 257 (1995).

[6] T. Narasimhamurti, Photoelastic and Electrooptic Properties of the Crystals, Mir, Moscow 1984, p. 621.

[7] I.I. Polovinko, S.A. Sveleba, V.S. Zhmurko, J. Stankowski, Z. Trybula, W. Kempinski, Phys. Status Solidi A 135, 527 (1993).

[8] S. Sveleba, V. Zhmurko, V. Kapustianik, I. Polovinko, Z. Trybula, Phys. Status Solidi A 140, 573 (1993).

[9] Yu.A. Izyumov, V.M. Syromyatnikov, Phase Transitions and Cristals Symmetry, Nauka, Moskva 1984, p. 284 (in Russian).

[10] V.A. Golovko, D.G. Sannikov, J. Phys., Condens. Matter 1, 5497 (1989).

[11] V.A. Golovko, A.P. Levanyuk, Fiz. Tverd. Tela 23, 3179 (1981).

[12] A.S. Sonin, A.S. Vasilevskaya, Electrooptic Crystals, Atomisdat, Moskva 1971, p. 327 (in Russian).

[13] S.A. Sveleba, I.I. Polovinko, Yu.I. Pankivskyi, V.A. Sergatyuk, M.I. Bublyk, V.I. Mokryi, Ukrainian Phys. J. 42, 294 (1997). 